\title{
Indicação de exodontias e fatores associados: estudo transversal na população indígena Kiriri
}

\author{
Recommendation of tooth extraction and associated factors: \\ cross-sectional study in the Kiriri indigenous population
}

Tayane da Rocha Costa Coelho (https://orcid.org/0000-0003-2720-2537) ${ }^{1}$

Henrique Bonione Carneiro Sampaio (https://orcid.org/0000-0001-8243-6278) ${ }^{1}$

Nara Santos Araujo (https://orcid.org/0000-0002-1166-8300) ${ }^{1}$

Patricia Ramos Cury (https://orcid.org/0000-0001-8907-0483) ${ }^{2}$
${ }^{1}$ Programa de Pós-

Graduação em Odontologia e Saúde, Faculdade de

Odontologia, Universidade Federal da Bahia (UFBA). Av. Araújo Pinho 62, Canela. 40110-150 Salvador BA Brasil.patricia.cury@ufba.br ${ }^{2}$ Departamento de Clínica Odontológica, Periodontia, Faculdade de Odontologia, UFBA. Salvador BA Brasil.

\begin{abstract}
The scope of this cross-sectional study was to evaluate the prevalence and the associated factors of the recommendation of dental extraction in the adult Kiriri Indigenous population of northeastern Brazil. A total of 225 natives $(\geq 19$ years) were evaluated. The extraction criteria were teeth with clinical attachment loss $\geq 50 \%$ in at least 3 sites, and residual roots. Statistical models were used to evaluate associations between the need for dental extraction and selected variables. The percentage of teeth recommended for dental extraction was $4.98 \%$, characterizing a mean of 1.24 tooth per individual. A regression analysis showed that age $\geq 35$ years $(O R=2.24$, 95\%CI: 1.13-4.43, $p=0.02)$, income $<R \$ 570.00$ $(O R=3.34,95 \% C I: 1.19-9.37, p=0.02)$ and plaque index $\geq 40 \%$ (OR=2.38, 95\%CI: 1.24-4.56, $p=0,01)$ were significantly associated with indication for dental extraction. A prevalence of 33\% of subjects with dental extraction recommendation were associated with age older than 35 years, plaque index $\geq 40 \%$ and income less than a minimum wage were related with the recommendation for extraction. This study reflects the importance of prioritizing oral health prevention, as well as the need to expand complex dental services.

Key words Tooth extraction, Tooth loss, South American Indians, Periodontal diseases, Risk factors
\end{abstract}

Resumo $O$ objetivo deste artigo foi avaliar a prevalência e os fatores associados à indicação de exodontia em uma população adulta de indios Kiriri do Nordeste do Brasil. 225 indígenas ( $\geq 19$ anos) foram avaliados. Os critérios de indicação de exodontia adotados foram: dentes com perda de inserção clínica $\geq 50 \%$, em pelo menos 3 sítios, $e$, também, raízes residuais. Modelos estatísticos foram utilizados para avaliar associações entre necessidade de exodontia e as variáveis selecionadas. A porcentagem de dentes com indicação de exodontia foi de 4,98\%, caracterizando uma média de 1,24 dente por indivíduo. A análise de regressão mostrou associação positiva entre indicação de exodontia e idade $\geq 35$ anos $(O R=2,24$, 95\%IC: 1,13-4,43, $p=0,02)$, renda $<R \$ 570,00$ $(O R=3,34,95 \% I C: 1,19-9,37, p=0,02)$ e indice de placa $\geq 40 \%$ (OR=2,38, 95\%IC: 1,24-4,56, $p=0,01)$. Uma prevalência de $33 \%$ de indivíduos com indicação de um ou mais dentes para exodontia está, principalmente, relacionada aos fatores de risco: idade maior que 35 anos, indice de placa $\geq 40 \%$ e renda inferior a um salário mínimo. Esse estudo reflete tanto a importância da priorização da prevenção em saúde bucal, como a necessidade de ampliação do acesso aos serviços de maior complexidade.

Palavras-chave Extração dentária, Perda de dente, Indios Sul-Americanos, Doenças periodontais, Fatores de risco 


\section{Introdução}

A saúde bucal é um componente fundamental para o bem-estar físico e mental. Neste contexto, a perda de dentes permanentes reflete o efeito cumulativo de doenças e fatores socias e pode ser considerada um importante indicador de vigilância em saúde bucal usado para sua monitoração global a nível da população $0^{1-4}$.

A perda de dentes permanentes é um processo multifatorial que, relacionado às doenças bucais, está associado principalmente à cárie dentária, ao trauma e à doença periodontal ${ }^{3-5}$. $\mathrm{O}$ status de perda dentária pode afetar a qualidade de vida através de diferentes caminhos. Primeiro, pode influenciar adversamente na funcionalidade, repercutindo nas atividades diárias dos indivíduos afetados, como a mastigação, a escolha de alimentos e a fala ${ }^{1}$. Em segundo lugar, o comprometimento estético pode prejudicar a autoestima e as relações sociais ${ }^{2}$.

As desigualdades socioeconômicas e culturais, as crenças, $\mathrm{o}$ acesso ao atendimento, além da própria percepção individual, contribuem para esta condição e exercem grande influência sobre a decisão de extrair um dente ${ }^{6-8}$. Por sua vez, fatores como tabagismo, presença de doenças sistêmicas, nível de escolaridade, renda mensal, faixa etária, sexo, estado civil e raça, são considerados fatores modificativos ${ }^{9-12}$.

Estudos realizados com adultos brasileiros relatam que a perda dentária é mais prevalente em indivíduos residentes na zona rural, do sexo feminino, idosos, de baixa renda, com menor grau de escolaridade e usuários do serviço público de saúde $^{13,14}$. Esse cenário se agrava ao evidenciar que, embora o Brasil se destaque na produção científica, parcelas da sociedade geralmente não são incluídas nesses estudos, como é o caso das populações indígenas.

De acordo com os resultados do Censo Demográfico 2010, realizado pelo Instituto Brasileiro de Geografia e Estatística (IBGE) ${ }^{15}$, 817,9 mil pessoas se declararam indígenas, representando $0,4 \%$ da população total do Brasil, das quais $63,8 \%$ viviam na área rural, desses $57,7 \%$ moravam em Terras Indígenas oficialmente reconhecidas. No tocante ao contexto territorial, o Censo retratou que na região Nordeste os indígenas eram mais representativos fora do território indígena (54,4\%).

No Nordeste brasileiro, segundo dados publicados em 2014 ${ }^{16}$, os indígenas Kiriri constituem uma população rural com aproximadamente 2.498 índios residentes em reservas isoladas, no estado da Bahia, mais especificamente nos municípios de Banzaê (aldeias: Baixa da Cangalha, Araçá, Baixa do Juá, Cajazeiras, Marcação, Mirandela, Pau Ferro, Segredo, Lagoa Grande e Tuxá), Muquém de São Francisco (aldeia Itaim) e Quijingue (aldeia Baixa da Cangalha) (Figura 1). Esta limitação geográfica associada à falta de ajuda com o transporte, atendimento odontológico público limitado e alto custo de atendimento privado, restringem o acesso a serviços odontológicos. Enquanto hábitos culturais em relação a cuidados de saúde, como uso de medicamentos à base de plantas e orações, são tradições mantidas.

Diante deste contexto, influências externas existem, porém, são limitadas. Dessa maneira, a população conseguiu manter parte do seu estilo de vida social, comportamental e cultural preservados. Quanto aos hábitos alimentares, a refeição é composta basicamente de alimentos provindos de agricultura familiar local, como feijão, farinha de mandioca e o milho, além de frutas de produção local (especialmente caju, manga, seriguela e pinha), as verduras mais encontradas são maxixe, quiabo e abóbora; carnes não são acessíveis a todos. Produtos industrializados são consumidos por pequena parcela, porém crescente, da população ${ }^{17}$.

Até a década de 1970, os dados epidemiológicos das condições de saúde bucal na população indígena eram escassos. A maioria dos estudos realizados no Brasil ${ }^{14,18-21}$ relatam o índice de dentes permanentes cariados, perdidos e obturados (CPO-d). Os estudos pré-existentes concordam que existe um déficit no quesito instrução em higiene bucal e acesso a tratamento odontológi$\mathrm{Co}^{14,18-22}$. Alta prevalência de cárie ${ }^{14,18-20} \mathrm{e}$ grande comprometimento periodontal ${ }^{14,20}$ também são relatados. Entretanto, não há estudos que contemplem a indicação de extração, e os possíveis fatores de risco associados, em populações indígenas brasileiras, especificamente na região nordeste do Brasil.

O presente estudo parte da premissa de que o conhecimento obtido por intermédio da produção científica sobre a saúde das etnias indígenas do Brasil pode ser útil para a sua melhor compreensão. A realização de novos levantamentos epidemiológicos pode subsidiar tanto a tomada de decisão quanto os rumos da própria ciência e de políticas científicas e tecnológicas voltadas para a saúde indígena. Em face do exposto, o objetivo do presente estudo foi avaliar a prevalência da indicação de exodontia, de acordo com critérios periodontais e de destruição coronária, e associá -la com os possíveis fatores de risco relacionados 


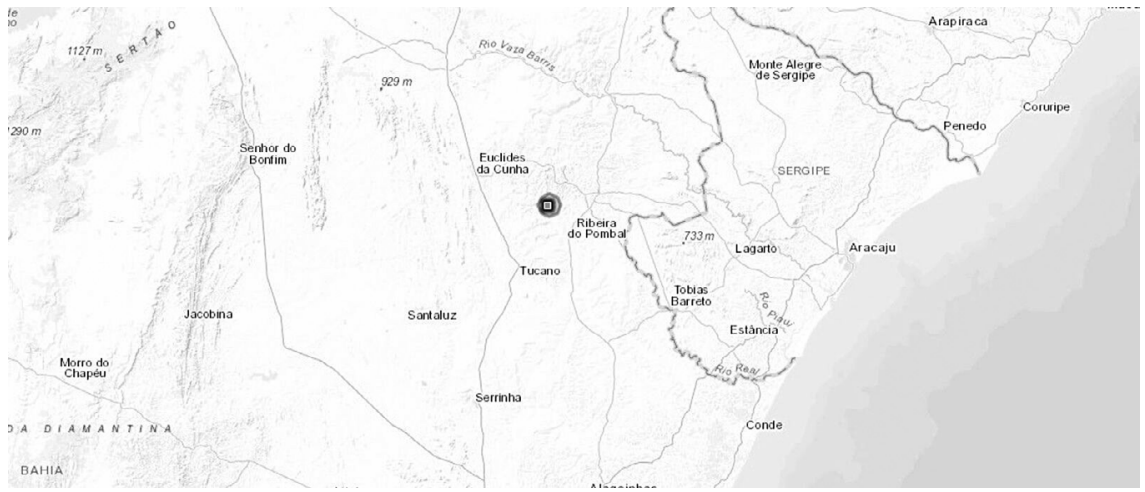

Figura 1. Mapa da Terra Indígena Kiriri.

Fonte: Terra indígena Kiriri [Homepage da Internet]. Terras indígenas [acesso em 17 de set de 2021]. Disponível em: https:// terrasindigenas.org.br/pt-br/terras-indigenas/3732

a essa indicação, em uma população adulta de índios Kiriri.

\section{Materiais e métodos}

O presente estudo foi conduzido de acordo com a Declaração de Helsinki da Associação Médica Mundial e teve sua aprovação pela Comissão Nacional de Ética em Pesquisa do Ministério da Saúde, Brasília, Brasil. Autorização para a sua realização foi obtida junto à Fundação Nacional de Saúde (FUNASA), pelo Distrito de Saúde Especial Indígena (DSEI) e pela Fundação Nacional do Índio (FUNAI). Todos os participantes assinaram um Termo de Consentimento Livre e Esclarecido.

\section{Delineamento do estudo e Amostragem}

Esse estudo de caráter analítico e transversal utilizou uma amostra da população de adultos $(\geq 19$ anos de idade) indígenas Kiriri, calculada com base nos dados fornecidos pela FUNASA em 2011.

À época da pesquisa, entre 2011 e 2012, dos 2.182 índios Kiriri que viviam em 10 diferentes aldeias na reserva indígena do município de Banzaê, 1.025 eram adultos. Erro de amostragem de $5 \%$ e intervalo de confiança de $95 \%$ foram considerados para o cálculo da amostra. Considerando a falta de informações sobre a prevalência da doença periodontal na população alvo, foi assumida uma taxa de $79 \%$ baseada na prevalência da per- da do nível de inserção clínica (NIC) $\geq 5$ milímetros relatada para brasileiros ${ }^{23}$. Foram considerados critérios de exclusão, a ausência completa de dentes e a presença de doenças cardiovasculares ou outras condições que requeressem o uso de antibióticos antes do exame periodontal. Como critério de inclusão, considerou-se indivíduos de ambos os sexos e com idade $>19$ anos. Levandose em consideração uma taxa de resposta de $90 \%$, a amostra calculada foi de 205 indivíduos.

Considerando $10 \%$ de perda, 226 indivíduos foram selecionados aleatoriamente, a partir de uma lista de indivíduos adultos fornecidos pela FUNASA, e convidados a participar do estudo. As autoridades indígenas locais encorajaram a comunidade a participar. Alguns dias anteriormente ao exame clínico, profissionais envolvidos na pesquisa visitaram os índios selecionados e explicaram o objetivo do estudo. A taxa de resposta foi de $99,6 \%$, um selecionado era completamente desdentado e, portanto, foi excluído da análise. Desta forma, 225 indivíduos com idade variando de 19 a 77 anos foram examinados.

\section{Procedimento operacional no campo}

O trabalho de campo foi realizado no ano de 2011. Antes do exame clínico, entrevistas presenciais, mediante a aplicação de questionário estruturado, contendo informações sobre o status demográfico (idade, sexo e escolaridade), socioeconômico (renda individual) e de saúde (tabagismo, diabetes e ida ao dentista), foram realizadas por 2 enfermeiras. O teste da glicemia em jejum 
foi realizado em todos os indivíduos utilizando medidor de glicemia padronizado (OneTouch Ultra Mini, LifeScan, Milpitas, CA, EUA).

\section{Avaliação Periodontal}

Os exames clínicos foram realizados em 10 locais, incluindo escolas e instalações de saúde. $\mathrm{O}$ exame clínico contou com o auxílio de uma lanterna (Turboled, Nautika, São Paulo-SP, Brasil) e os participantes mantinham-se sentados em uma cadeira regular. Quatro periodontistas, assistidos por 04 alunos de graduação da Faculdade de Odontologia da Universidade Federal da Bahia (FOUFBA), realizaram os exames.

Previamente, os examinadores foram treinados para a exatidão e repetitividade de uma população de 10 indivíduos não relacionados ao estudo. Os coeficientes de correlação intraclasse e interclasse (CCI) foram calculados e revelaram a reprodutibilidade intra e inter-examinador ${ }^{24}$. Para a profundidade de sondagem (PS) foram obtidos os resultados intra-examinador: CCI 0.93-0.95 e inter-examinador: CCI $\geq 0.81$. Para a distância entre a junção cemento-esmalte e a gengiva marginal livre (JCE-GML) encontrou-se intra-examinador: CCI 0.81-0.83 e inter-examinador: $\mathrm{CCI} \geq 0.75$.

Todos os dentes permanentes totalmente irrompidos, excluindo-se os terceiros molares, foram examinados usando uma sonda periodontal manual (PCP -UNC 15, Hu-Friedy, Chicago, IL, EUA). A PS e a distância JCE-GML foram medidas em seis sítios por dente (mésio-vestibular, médio-vestibular, disto-vestibular, disto-lingual, médio-lingual e mésio-lingual). As medições foram feitas em milímetros e arredondado para o próximo milímetro inteiro. O NIC foi calculado com a soma das medições entre PS e JCE-GML. O Índice de Placa Visível (IPV) ${ }^{25}$ foi avaliado durante o exame clínico. O valor do IPV foi obtido em porcentagem, somando-se o número de superfícies com placa visível, dividindo-se pelo total de superfícies examinadas e o resultado obtido foi multiplicado por $100^{26}$.

\section{Análise de dados}

$\mathrm{Na}$ análise estatística, o percentual de indivíduos com pelo menos um dente com indicação de exodontia foi calculado. A análise dos dados foi realizada utilizando o SPSS versão 13.0 (SPSS Inc., Chicago, IL, EUA).

A condição demográfica, socioeconômica e dados relacionados com a saúde foram categori- zados. A idade foi dividida em 2 categorias: 19 a 34 anos e $\geq 35$ anos, para análise da prevalência, tanto na análise bivariada quanto no cálculo da regressão logística. O nível de escolaridade foi categorizado como $<9$ anos de escolaridade ou $\geq 9$ anos de escolaridade. $\mathrm{O}$ estado econômico foi categorizado como: renda mensal $<\mathrm{R} \$ 570,00$ ou $\geq \mathrm{R} \$ 570,00$, sendo esse o valor correspondente ao salário mínimo à época da pesquisa. Os participantes foram classificados como não-fumantes ou fumantes e questionados quanto ao número de cigarros, charutos ou cachimbos fumados ao dia.

Participantes eram classificados como diabéticos caso houvesse o auto relato ou a glicemia de jejum detectada fosse $\geq 126 \mathrm{mg} / \mathrm{dL}$ associado com sintomas de diabetes (polidipsia, poliúria e perda de peso não explicada). Caso não apresentassem nenhum desses critérios os indivíduos eram classificados como não diabéticos.

O critério de indicação de exodontia usado baseou-se nas normas publicadas por Zitzmann et al. ${ }^{26}$ que pontuaram como principal parâmetro a quantidade de osso remanescente, avaliado pelo nível de inserção clínica (NIC), verificando-se 06 sítios em cada dente, sendo 03 em cada face livre, conforme relatado anteriormente. Dentes com perda de inserção clínica $\geq 50 \%$ em pelo menos 3 sítios examinados e raízes residuais foram indicados para a exodontia. Em sequência, a indicação de extração de cada indivíduo foi categorizada em uma planilha, para posterior análise estatística.

Estatística descritiva foi usada para avaliar a indicação de exodontia de acordo com os fatores de risco, relacionados a aspectos sociais e de saúde geral, já descritos na literatura (idade, sexo, educação, renda, tabagismo e estado diabético). O teste Qui-quadrado foi utilizado para comparar todos os indivíduos em uma análise bivariada com a indicação de extração e as outras todas variáveis supracitadas.

Regressão logística foi empregada para determinar o conjunto de variáveis que foram associados com a indicação de extração. Sexo, idade, tabagismo, diabetes, escolaridade, índice de placa e renda foram firmados no modelo. O Odds Ratio (OR) juntamente com o intervalo confiança de $95 \%$ foram calculados. O nível escolhido de significância foi de $5 \%$.

\section{Resultados}

A Tabela 1 descreve as características gerais da população avaliada. Registrou-se um total de 
1.150 dentes ausentes, com média de 5,1 dentes ausentes por indivíduo. Em relação aos dentes presentes, $280(4,98 \%)$ apresentavam indicação para exodontia, correspondendo a uma média de 1,24 dente por indivíduo. Obteve-se uma prevalência de $33 \%$ de indivíduos com indicação de um ou mais dentes para exodontia. Pouco mais da metade da população avaliada $(50.7 \%)$ era fumante, com uma média de 3,7 cigarros, cachimbos ou charutos fumados ao dia.

A análise bivariada (Tabela 2), mostrou que a indicação para exodontia em dentição permanente está significativamente associada à idade $\geq 35$ anos $(\mathrm{n}=51,1 \%, \mathrm{OR}=3,57, \mathrm{IC} 95 \%=2,00$ $6,38, \mathrm{p}<0,001)$, ao elevado IP $(\mathrm{n}=49,4 \%$, $\mathrm{OR}=3,04, \mathrm{IC} 95 \%=1,71-5,40, \mathrm{p}<0,001)$, à baixa renda $(n=36,9 \%, O R=2,92$, IC $95 \%=1,15-7,37$, $\mathrm{p}=0,01)$ e baixo nível de escolaridade $(\mathrm{n}=39,7 \%$, $\mathrm{OR}=2,74, \mathrm{IC} 95 \%=1,38-5,43, \mathrm{p}=0,003)$.

A análise de regressão logística (Tabela 3) revelou que a probabilidade de um dente perma-

Tabela 1. Características da população do estudo (Índios Kiriri, Brasil; N=225).

\begin{tabular}{|c|c|c|}
\hline \multirow[b]{2}{*}{ Características } & \multicolumn{2}{|c|}{ Índios Kiriri } \\
\hline & $\mathbf{N}$ & $\begin{array}{c}\text { Percentual } \\
\text { (\%) }\end{array}$ \\
\hline \multicolumn{3}{|l|}{ Sexo } \\
\hline Feminino & 124 & 55,1 \\
\hline Masculino & 101 & 44,9 \\
\hline \multicolumn{3}{|l|}{ Idade (anos) } \\
\hline$<35$ & 137 & 60,9 \\
\hline$\geq 35$ & 88 & 39,1 \\
\hline \multicolumn{3}{|l|}{ Escolaridade } \\
\hline$<9$ anos & 156 & 70 \\
\hline$\geq 9$ anos & 67 & 30 \\
\hline \multicolumn{3}{|l|}{ Renda } \\
\hline$<\mathrm{R} \$ 570,00$ & 187 & 83,9 \\
\hline$\geq \mathrm{R} \$ 570,00$ & 36 & 16,1 \\
\hline \multicolumn{3}{|l|}{ Tabagismo } \\
\hline Não & 111 & 49,3 \\
\hline Sim & 114 & 50,7 \\
\hline \multicolumn{3}{|l|}{ Diabetes } \\
\hline Não & 211 & 93,7 \\
\hline $\operatorname{Sim}$ & 14 & 6,3 \\
\hline \multicolumn{3}{|l|}{ Índice de Placa (IP) } \\
\hline$<40 \%$ & 140 & 62,2 \\
\hline$\geq 40 \%$ & 85 & 37,8 \\
\hline \multicolumn{3}{|l|}{ Ida ao Dentista } \\
\hline $\operatorname{Sim}$ & 204 & 91,0 \\
\hline Não & 21 & 9,0 \\
\hline
\end{tabular}

nente apresentar necessidade de exodontia foi 2,24 vezes mais alta nos participantes com idade $\geq 35$ anos (IC 1,13-4,43, $\mathrm{p}=0,02$ ). Aqueles com IP $\geq 40 \%$ apresentaram 2,38 vezes maiores chances (IC 1,24-4,56, p=0,009), assim como os participantes que possuíam menor renda, os quais mostraram um risco 3,34 vezes maior (IC 1,19$9,37, \mathrm{p}=0,02)$.

\section{Discussão}

No presente estudo, foi avaliada a prevalência de indicação de exodontia juntamente aos fatores associados em uma população adulta de índios Kiriri.

Os resultados demonstraram que 4,98\% dos dentes avaliados foram indicados à exodontia, o que corresponde a uma prevalência de 33\% da população avaliada e uma média de 1,24 dente por indivíduo. No entanto, uma média de 5,1 dentes por indivíduo já haviam sido extraídos no momento da avaliação. Um estudo realizado com Nativos do Alaska e índios americanos ${ }^{27}$ constatou uma média de 03 dentes indicados para extração por indivíduo. Em contrapartida, outro estudo brasileiro que avaliou as necessidades em saúde bucal dos índios Potiguara ${ }^{18}$ registrou resultados semelhantes ao do presente estudo, com uma média de 01 dente indicado para extração, por indivíduo, entre 35 e 44 anos; porém 22\% dos dentes permanentes já haviam sido submetidos à exodontia. Ambos os achados na população brasileira, caracterizam uma abordagem tipicamente cirúrgica, frente às patologias bucais. Por outro lado, um estudo realizado no Parque Indígena Xingu avaliou a proporção do número de exodontias em relação a procedimentos menos invasivos e constatou uma redução considerável dessa proporção com o passar dos anos (20042008), o que reflete uma melhora no cenário de prevenção e cura durante o período citado; os autores associam essa melhora a uma parceria entre a Universidade de São Paulo e o DSEI (Distrito Sanitário Indígena) da região ${ }^{28}$.

Idade elevada foi um dos fatores associados mais relevantes quando se indicam extrações, enquanto o sexo não obteve relevância nesse estudo. A idade elevada foi, também, considerada como um fator relevante em estudos anteriores ${ }^{5,10,13,20}$. Tal fato pode estar associado à evolução da doença periodontal, configurando níveis de perda óssea mais elevados em pacientes mais velhos ${ }^{8,29}$. Estudos prévios diferem dos presentes achados quanto à influência da variável sexo ${ }^{30,31}$, pois de- 
Tabela 2. Resultado da análise bivariada demonstrando a associação entre a indicação de exodontia com as variáveis sexo, idade, escolaridade, renda, tabagismo, diabetes, Índice de Placa (IP) e ida ao dentista (Índios Kiriri, Brasil; $\mathrm{N}=225$ ).

\begin{tabular}{|c|c|c|c|c|c|}
\hline \multirow{2}{*}{ Variáveis } & \multicolumn{5}{|c|}{ Indicação de exodontia } \\
\hline & Não & Sim & OR & 95\%IC & $\mathbf{P}$ \\
\hline Sexo & & & 1,16 & $0,66-2,02$ & 0,59 \\
\hline Feminino & $67,7 \%$ & $32,3 \%$ & & & \\
\hline Masculino & $64,4 \%$ & $35,6 \%$ & & & \\
\hline Idade (anos) & & & 3,57 & $2,00-6,38$ & $<0,001$ \\
\hline 19-34 & $77,4 \%$ & $22,6 \%$ & & & \\
\hline$\geq 35$ & $48,9 \%$ & $51,1 \%$ & & & \\
\hline Escolaridade (anos) & & & 2,74 & $1,38-5,43$ & 0,003 \\
\hline$\geq 9$ & $80,6 \%$ & $19,4 \%$ & & & \\
\hline$<9$ & $60,3 \%$ & $39,7 \%$ & & & \\
\hline Renda & & & 2,92 & $1,15-7,37$ & 0,01 \\
\hline$\geq \mathrm{R} \$ 540,00$ & $83,3 \%$ & $16,7 \%$ & & & \\
\hline$<\mathrm{R} \$ 540,00$ & $63,1 \%$ & $36,9 \%$ & & & \\
\hline Tabagismo & & & 1,32 & $0,75-2,30$ & 0,32 \\
\hline Não & $69,4 \%$ & $30,6 \%$ & & & \\
\hline Sim & $63,2 \%$ & $44,7 \%$ & & & \\
\hline Diabetes & & & 2,10 & $0,70-0,24$ & 0,17 \\
\hline Não & $67,8 \%$ & $32,2 \%$ & & & \\
\hline Sim & $50 \%$ & $50 \%$ & & & \\
\hline Índice de Placa (IP) & & & 3,04 & $1,71-5,40$ & $<0,001$ \\
\hline$<40 \%$ & $75,7 \%$ & $24,3 \%$ & & & \\
\hline$\geq 40 \%$ & $50,6 \%$ & $49,4 \%$ & & & \\
\hline Ida ao dentista & & & 2,35 & $0,95-5,83$ & 0,06 \\
\hline Sim & $68,2 \%$ & $31,8 \%$ & & & \\
\hline Não & $47,6 \%$ & $52,4 \%$ & & & \\
\hline
\end{tabular}

Fonte: Elaborado pelos autores.

Tabela 3. Resultado da regressão logística considerando as variáveis idade, renda e índice de placa (IP) em relação à indicação de exodontia como variável dependente (Índios Kiriri, Brasil; N=225).

\begin{tabular}{|c|c|c|}
\hline \multirow{2}{*}{ Variáveis } & \multicolumn{2}{|c|}{ Indicação de exodontia } \\
\hline & OR (95\%IC) & $\mathbf{P}$ \\
\hline Idade (anos) & & 0,02 \\
\hline 19-34 & 1 & \\
\hline$>35$ & $2,24(1,13-4,43)$ & \\
\hline Renda & & 0,02 \\
\hline$\geq \mathrm{R} \$ 570$ & 1 & \\
\hline$<\mathrm{R} \$ 570$ & $3,34(1,19-9,37)$ & \\
\hline Índice de Placa (IP) & & 0,009 \\
\hline$\leq 40 \%$ & 1 & \\
\hline$>40 \%$ & $2,38(1,24-4,56)$ & \\
\hline
\end{tabular}

Fonte: Elaborado pelos autores. mostraram que mulheres possuem maiores necessidades de extrações dentárias; usualmente o sexo feminino é mais presente na participação de estudos e na busca por atendimento odontológico, no entanto, o presente estudo dispôs de uma amostra relativamente homogênea em relação ao sexo, sendo uma possível justificativa para os achados equilibrados. Esses resultados podem, também, ter associação com as idiossincrasias referentes à cultura indígena, resultando na indiferenciação entre os sexos quanto a hábitos de higiene e iniciativa para procura de tratamento odontológico.

Fatores econômicos foram anteriormente descritos como indicadores de risco para agravamento das condições de saúde bucal em populações indígenas ${ }^{32}$. Uma possível explicação para essa relação está relacionada a maiores possibilidades de acesso ao atendimento odontológico - para os indivíduos com melhor remuneração - 
devido aos custos que envolvem essa procura, seja no âmbito público ou privado. No presente estudo, indígenas que apresentavam renda inferior a R \$ 570,00 detiveram 2,92 vezes mais chances de possuir indicação de extração. Esses achados caracterizam uma realidade familiar à população brasileira, no que se refere a uma comunidade que apresenta hábitos culturais e sociais distintos da população não indígena, mas que também está sujeita às desigualdades socioeconômicas e suas repercussões na saúde. Na prática, tais achados representam a necessidade de melhoria no acesso e no atendimento em saúde odontológica que abranja as necessidades da população.

Os dados deste estudo retratam que indivíduos com escolaridade inferior a nove anos apresentaram 2,57 vezes maiores chances de ter indicação de extração. Um estudo ${ }^{33}$ realizado com indígenas norte-americanos sugeriu, após a contabilização de vários indicadores demográficos, que conhecimento, crenças e barreiras relacionadas à saúde podem ter um papel importante em comportamentos de saúde bucal. A educação das mães (alta) e estar empregado foram positivamente associados a melhores escores de comportamento em saúde bucal. Níveis de escolaridade mais baixos usualmente refletem uma educação em saúde mais limitada; na esfera da saúde bu$\mathrm{cal}$, pode caracterizar restrições no conhecimento dos métodos de prevenção de doenças bucais, principalmente relacionados à higienização, bem como a busca tardia para tratamento odontológico.

Em relação ao tabagismo, observa-se controvérsias na literatura em relação a essa pesquisa. Alguns autores ${ }^{12,34}$ pontuaram em seus estudos que o hábito de fumar foi um fator de risco para doença periodontal e consequentemente perda dentária. Neste estudo, tanto na análise bivariada quanto na regressão logística o hábito de fumar não se relacionou com a perda dentária, no entanto, não é possível dissociá-la do hábito de fumar, pois o que dita se fumar é ou não um fator associado substancial é a quantidade que cada indivíduo consome por dia e quais substâncias estão sendo consumidas ${ }^{35}$. Uma possível explicação para os presentes achados é que, embora 50,7\% da população fossem fumantes, o número médio de cigarros, cachimbos ou charutos fumados por dia era de 3,7 , o que pode ser considerado como fumo leve $\mathrm{e}^{18}$. Um aspecto importante na presente população é a variação do tipo de fumo, sendo mais utilizados os cigarros fabricados a mão com diferentes produtos, bem como, os cachimbos. Além disso, muitos indivíduos relatam exercer o hábito de fumar apenas durante as cerimônias indígenas (diárias ou semanais).

Quanto ao diabetes, observando-se apenas o dado estatístico não foi encontrada significância, mas ao analisar a indicação de extração, metade dos pacientes que possuíam a doença apresentaram necessidade de exodontia. Tais achados estão de acordo com a literatura ${ }^{9,12}$. Logo, apesar da irrelevância estatística neste estudo e, pautado em dados publicados sobre periodontite na presente população ${ }^{36}$, sugere-se que o diabetes seja investigado em estudos futuros como um possível indicador de risco para a necessidade de exodontia.

A Secretaria Especial de Saúde Indígena (Sesai) coordena e executa a Política Nacional de Atenção à Saúde dos Povos Indígenas através do Subsistema de Atenção à Saúde Indígena, articulado com o SUS (SasiSUS). Foram criados, desde 1999, 34 DSEIs objetivando o desenvolvimento de ações de atenção em saúde aos povos indígenas $^{37}$. Os índios Kiriri estão cobertos para atendimento odontológico através do suporte do DSEI Bahia $^{38}$; sendo responsável pelo planejamento, oferta de serviços de saúde e visitação nas áreas cobertas. No entanto, à época da pesquisa, limitações de transporte e divergências culturais foram considerados fatores que reduziam a procura por atendimento odontológico. Diferentes estudos realizados com populações indígenas em todo o mundo enfatizam a necessidade de constituir-se serviços de saúde culturalmente sensíveis, em diferentes níveis de atenção à saúde, visando manter o respeito à cultura ao centro da relação entre pacientes e serviços de saúde ${ }^{39}$. Dessa maneira, evidencia-se uma necessidade de planejamento em atendimento mais efetivo, também associado ao deslocamento do consultório odontológico até o território indígena com o objetivo de cobrir limitações logísticas de movimentação dos índios, além de estimular a busca por atendimento e tentar traduzir, nessas visitas, o respeito à cultura e às características do povo indígena durante o acompanhamento.

Frente aos resultados encontrados pelo presente estudo, se faz necessária uma abordagem mais efetiva de prevenção e tratamento das doenças bucais nesta população. Um estudo realizado na população de etnia Xukuru de Ororubá retratou resultados de autopercepção da saúde bucal incompatíveis com a realidade, onde a maioria dos indivíduos investigados, apesar da necessidade de intervenções odontológicas, relataram não apresentar impacto negativo da saúde bucal em suas vidas ${ }^{40}$. Nesse ínterim, é possível estabelecer um paralelo entre um estudo americano que ob- 
jetivou analisar a relação entre perda dentária e integração social em adultos mais velhos, descendentes próximos de variadas etnias. Como resultado, obteve-se uma associação positiva entre um maior número de dentes e uma melhor relação social. Dessa maneira, é possível levantar uma reflexão: as individualidades socioculturais das populações indígenas podem relacionar-se ao não reconhecimento da presença de um problema em saúde, bem como ao manejo inadequado após sua detecção. Nesse caso, embasado no presente estudo, táticas de informar e estimular hábitos de saúde bucal, bem como de agregar conhecimento quanto à necessidade de intervenção odontológica - dentro dos limites de respeito ao local físico de moradia, hábitos e cultura indígenas - passam a ser a principal ferramenta, se não de reversão, mas de prevenção das doenças bucais que possam se desenvolver em indicação de exodontia $^{41,42}$.

Outrossim, o processo saúde-doença do povo indígena pode, segundo suas crenças, ser relacionado a diferentes etiologias como alterações da alma, feitiçaria, explicações naturais, sobrenaturais, entre outras ${ }^{43}$. De maneira que, práticas tradicionais são postas em prática no intuito de reduzir a dor ou eliminar a doença como um todo, o que reflete em barreiras encontradas no manejo em saúde, relacionadas à necessidade de intervenção mínima nos costumes culturais indígenas, as quais são desafiadoras. Dados da literatura retratam alguns métodos os quais poderiam reduzir o nível destas dificuldades: respeito ao modelo de saúde já instituído na etnia; a cooperação, comunicação e orientação entre grupos de saúde que assistem os indígenas; participação ativa de índios locais na equipe de assistência; projetos em saúde bucal preconizados para longa duração ${ }^{44}$. Não obstante, o apoio político ocupa espaço indispensável ao desenvolvimento e maturação da qualidade e ampliação do atendimento em saúde bucal.

Este estudo serve de orientação para que políticas públicas visem, em todas as suas particularidades, as necessidades de grupos específicos, como os indígenas. Estratégias precisam ser elaboradas, de maneira a otimizar a educação em saúde bucal e implementar acesso a tratamento odontológico qualificado à população brasileira em toda sua extensão e diversidade. O investimento na qualidade de vida e na saúde bucal de cada população irá refletir numa melhora do quadro de saúde geral da população brasileira.

Como limitação desse estudo é possível ressaltar a utilização, apenas, do nível clínico de inserção como parâmetro para indicação de exodontia - além de raízes residuais; no entanto, quando se planeja um tratamento é necessário se levar em conta todo aspecto funcional de cada indivíduo, como a condição da coroa, presença de cáries extensas, inclinações e extrusões dentárias e histórico médico-odontológico.

Conclui-se que, com uma prevalência de 33\% de indivíduos com indicação de um ou mais dentes para exodontia, os fatores de risco mais relacionados à essa indicação foram idade maior que 35 anos, índice de placa $\geq 40 \%$ e renda inferior a um salário mínimo. Esses resultados claramente refletem a necessidade de priorizar a dimensão preventiva e educativa, mas também de ampliação do serviço odontológico de maior complexidade e um maior planejamento em atendimento, de maneira a suprir as necessidades de intervenção com procedimentos eficazes. De caráter complementar, porém impactante no grupo em estudo, sugere-se a capacitação e participação direta de indígenas no planejamento das práticas de saúde operadas pela equipe de saúde bucal. 


\section{Colaboradores}

HBC Sampaio foi responsável pela concepção do estudo, coleta e análise dos dados. PR Cury participou da análise dos dados e supervisão de todo o processo. TRC Coelho e NS Araujo contribuíram com a redação do artigo.

\section{Referências}

1. Brennan DS1, Spencer AJ, Roberts-Thomson KF. Tooth loss, chewing ability and quality of life. Qual Life Res 2008; 17(2):227-235.

2. Craddock HL. Consequences of tooth loss (Part 1): The patient perspective--aesthetic and functional implications. SADJ 2010; 65(6):242,244-245.

3. Grimoud AM, Lucas S, Sevin A, Georges P, Passarrius $\mathrm{O}$, Duranthon F. Frequency of dental caries in four historical populations from the chalcolithic to the middle ages. Int J Dent 2011; 2011:519691.

4. Lang NP, Tonetti MS. Periodontal diagnosis in treated periodontitis. Why, when and how to use clinical parameters. J Clin Periodontol 1996; 23(3 Pt 2):240-250.

5. Tiwari T, Scarbro S, Bryant LL, Puma J. Factors Associated with Tooth Loss in Older Adults in Rural Colorado. J Community Health 2016; 41(3):476-481

6. Song IS, Han K, Choi YJ, Ryu JJ, Park JB. Influence of oral health behavior and sociodemographic factors on remaining teeth in Korean adults: 2010-2012 Korea national health and nutrition examination survey. Medicine (Baltimore) 2016; 95(48):e5492.

7. Goulart MA, Vettore MV. Is the relative increase in income inequality related to tooth loss in middle-aged adults? J Public Health Dent 2016; 76(1):65-75.

8. Figueiredo A, Soares S, Lopes H, Santos JN, Ramalho LM, Cangussu MC, Cury PR. Destructive periodontal disease in adult Indians from Northeast Brazil: cross-sectional study of prevalence and risk indicators. $J$ Clinical Periodontol 2013; 40(11):1001-1006.

9. Presson SM, Niendorff WJ, Martin RF. Tooth Loss and Need for Extractions in American Indian and Alaska Native Dental Patients. J Public Health Dentistry 2000; 60(1):267-272.

10. Chrysanthakopoulos NA. Periodontal Reasons for Tooth Extraction in a Group of Greek Army Personnel. J Dental Res Dental Clinics Dental Prospects 2011; 5(2):55-60.

11. Jovino-Silveira RC, Caldas AF Jr, Souza EH, Gusmão ES. Primary Reason for Tooth Extraction in a Brazilian Adult Population. Oral Health Prev Dent 2005; 3:151-157.

12. Ong G. Periodontal disease and tooth loss. Int Dental $J$ 1998; 48(3):233-238.

13. Haikal DS, Martins AM, Aguiar PH, Silveira MF, Paula AM, Ferreira EF. Access to information on oral hygiene and tooth loss due to caries among adults. Cien Saude Colet 2014; 19(1):287-300.

14. Alves Filho P, Santos RV, Vettore MV. Factors associated with dental caries and periodontal diseases in Latin American indigenous peoples: a systematic review. Rev Panam Salud Publica 2014; 35(1):67-77.

15. Instituto Brasileiro de Geografia e Estatística (IBGE). Censo Demográfico 2010 - Características Gerais dos Indígenas - Resultados do Universo. Rio de Janeiro: IBGE; 2010.

16. Brasil. Ministério da Saúde (MS). Relatório de Dados Populacionais. Saúde Indígena [Internet]. [acessado 2018 nov 18]. Disponível em: http://portalms.saude. gov.br/saude-indigena.

17. Pacheco SSM. Interdições alimentares em situações de liminaridade entre os índios Kiriri do sertão da Bahia. Espaço Ameríndio 2011; 5(1):57-68. 
18. Sampaio FC, Freitas CH, Cabral MB, Machado AT. Dental caries and treatment needs among indigenous people of the Potiguara Indian reservation in Brazil. Rev Panam Salud Publica 2010; 27(4):246-251.

19. Mauricio HA, Moreira RS. Oral health status of the ethnic group Xukuru from Ororubá: multilevel analysis. Rev Bras Epidemiol 2014; 17(3):787-800.

20. Ulhôa Netto E, Ferreira TFL, Drummond MM, Sanchez HF. Tooth loss and need of denture in Pataxó Natives. Rev Gaucha Odontol 2012; 60(2):195-201.

21. Vieira EMM, Ciesielski FIN, Gaetti-Jardim EC, Hespanhol D, Castro EVFL, Castro AL, Schweitzer CM, Gaetti-Jardim Junior E. Evaluation of oral health in a community of native brazilians of the Umutina Reservation, Mato Grosso State. Int J Odontostomatol 2011; 5(1):59-63.

22. Vieira EP, Barbosa MS, Quintão CC, Normando D. Relationship of tooth wear to chronological age among indigenous Amazon populations. PLoS One 2015; 10(1):e0116138.

23. Organização Mundial da Saúde (OMS). Levantamento epidemiológico básico de saúde bucal. $3^{\text {a }}$ ed. São Paulo: Santos; 1991.

24. Susin C, Dalla Vecchia CF, Oppermann RV, Haugejorden O, Albandar JM. Periodontal attachment loss in an urban population of Brazilian adults: effect of demographic, behavioral, and environmental risk indicators. J Periodontol 2004; 75:1033-1041.

25. Silness J, Löe H. Periodontal disease in pregnancy. II. Correlation between oral hygiene and periodontal condition. Acta Odontol Scand 1964; 22:121-135.

26. Zitzmann NU, Krastl G, Hecker H, Walter C, Waltimo T, Weiger R. Strategic considerations in treatment planning: deciding when to treat, extract, or replace a questionable tooth. J Prosthet Dent 2010; 104(2):8091.

27. Batliner T, Wilson A, Davis E, Gallegos J, Thomas J, Tiwari T, Fehringer K, Wilson K, Albino J. A Comparative Analysis of Oral Health on the Santo Domingo Pueblo Reservation. J Community Health 2016; 41(3):535-540.

28. Lemos PN, Rodrigues DA, Frazão P, Hirooka LB, Guisilini AC, Narvai PC. Atenção à saúde bucal no Parque Indígena do Xingu, Brasil, no período de 2004-2013: um olhar a partir de indicadores de avaliação. Cad Saude Publica 2018; 34(4):e00079317.

29. Renvert S, Persson RE, Persson GR. Tooth loss and periodontitis in older individuals: results from the Swedish national study on aging and care. J Periodontol 2013; 84:1134-1144.

30. Russell SL, Gordon S, Lukacs JR, Kaste LM. Sex/Gender Differences in Tooth Loss and Edentulism. Dent Clin North Am 2013; 57(2):317-337.

31. Peres MA, Barbato PR, Reis SC, Freitas CH, Antunes JL. Tooth loss in Brazil: analysis of the 2010 Brazilian Oral Health Survey. Rev Saude Publica 2013; 47(3):7889.

32. Arantes R, Santos RV, Coimbra Jr, CEA. Saúde oral entre os índios Xavante em Pimentel Barbosa, Mato Grosso, Brasil. Cad Saude Publica 2001; 17(2):375384.
33. Heaton B, Crawford A, Garcia RI, Henshaw M, Riedy CA, Barker JC. Oral health beliefs, knowledge, and behaviors in Northern California American Indian and Alaska Native mothers regarding early childhood caries. J Public Health Dentistry 2017; 77(4):350-359.

34. Krall EA, Dawson-Hughes B, Garvey AJ, Garcia RI. Smoking, smoking cessation, and tooth loss. J Dent Res 1997; 76(10):1653-1659.

35. Müller S, Eickholz P, Reitmeir P, Eger T. Long-term tooth loss in periodontally compromised but treated patients according to the type of prosthodontic treatment. A retrospective study. J Oral Rehabilitation 2013; 40:358-367.

36. Figueiredo A, Soares S, Lopes H, Santos JN, Ramalho LMP, Cangussu MC, Cury PR. Destructive periodontal disease in adult Indians from Northeast Brazil: cross-sectional study of prevalence and risk indicators. J Clinical Periodontol 2013;40(11):1001-1006.

37. Brasil. Ministério da Saúde (MS). Secretária Especial de Saúde Indígena [Internet]. [acessado 2019 ago 12]. Disponível em: http://www.saude.gov.br/sesa.

38. Brasil. Ministério da Saúde (MS). Distritos Sanitários Especiais Indígenas (DSEIs). Saúde Indígena [Internet]. [acessado 2018 nov 18]. Disponível em: http:// portalms.saude.gov.br/saude-indigena.

39. Cunha MA, Lino PA, Santos TR, Vasconcelos M, Lucas SD, Abreu MH. A 15-Year Time-series Study of Tooth Extraction in Brazil. Medicine (Baltimore) 2015; 94(47):e1924.

40. Mauricio HA, Moreira RS. Oral health status of the ethnic group Xukuru from Ororubá: multilevel analysis. Rev Bras Epidemiol 2014; 17(3):787-800.

41. Gomes SC, Esperidião MA. Indigenous peoples' access to health services in Cuiabá, Mato Grosso State, Brazil. Cad Saude Publica 2017; 33(5):e00132215.

42. Arcur TA, Chen H, Savoca MR, Anderson AM, Leng X, Bell RA, Quandt SA. Ethnic Variation in Oral Health and Social Integration among Older Rural Adults. $J$ Appl Gerontol 2013; 32(3):302-323.

43. Menta SA. Processo saúde-doença entre populações indígenas brasileiras: uma questão conceitual e instrumental. Tellus 2002; 2(2):2359-1943.

44. Tsai C, Blinkhorn A, Irving M. Oral Health Programmes in Indigenous Communities Worldwide-Lessons learned from the field: A qualitative systematic review. Community Dent Oral Epidemiol 2017; 45(5):389-397.

Artigo apresentado em 01/12/2018

Aprovado em 13/10/2019

Versão final apresentada em 15/10/2019

Editores-chefes: Romeu Gomes, Antônio Augusto Moura da Silva 